\title{
Prevalence of cigarette smoking in Iranian adolescents and young adults: a systematic review and meta-analysis
}

Mohammad Aryaie, ${ }^{1}$ Hamid Sharifi, ${ }^{1}$ Zahra Khorrami, ${ }^{1}$ Gelayol Ardalan, ${ }^{2}$ Leila Rajaei, ${ }^{2}$ Gholamreza Heydari ${ }^{3}$ and Armita Shahesmaeili ${ }^{1}$

${ }^{1} \mathrm{HIV} / \mathrm{STI}$ Surveillance Research Center, and WHO Collaborating Center for HIV Surveillance, Institute for Futures Studies in Health, Kerman University of Medical Sciences, Kerman, Islamic Republic of Iran (Correspondence to: A. Shahesmaeili: a.shahesmaeili@kmu.ac.ir). ${ }^{2}$ Adolescent and Youth Heath Office, Ministry of Health and Medical Education, Tehran, Islamic Republic of Iran. ${ }^{3}$ Tobacco Prevention \& Control Research Center, National Research Institute of TB \& Lung Diseases, Shahid Beheshti University of Medical Sciences, Tehran, Islamic Republic of Iran.

\begin{abstract}
Background: Cigarette smoking is a shared risk factor for a variety of health conditions.

Aims: To estimate the prevalence of cigarette smoking among Iranian adolescents and young adults.

Methods: In this systematic review and meta-analysis, we searched PubMed, Web of Science, Embase, Scopus and domestic databases from January 2001 to December 2018. Cross-sectional studies that reported the prevalence of cigarette smoking among high school students, university students or general population aged 14-30 years were eligible. Data on prevalence of smoking, age and gender of subjects, sample size, date, and location of studies were extracted by 2 independent reviewers.

Results: We included 99 studies in the analysis. For high school students, the pooled life-time prevalence of cigarette smoking was $13 \%$ in girls and $23 \%$ in boys. For university students, the life-time prevalence was $11 \%$ in women and $33 \%$ in men. The pooled prevalence of current and regular smoking among high school boys was $9 \%$ and $5 \%$, respectively, compared with $3 \%$ and $1 \%$ in girls. The pooled prevalence of current and regular smoking among male university students was $22 \%$ and $11 \%$, respectively, compared with $5 \%$ and $1 \%$ among female students. The prevalence of current smoking among the general population aged 15-24 years was 3.3-13.9\%. The prevalence of regular smoking among this population was $1.0-10.2 \%$.

Conclusion: Cigarette smoking is notable among Iranian young adults especially boys and university students. Our findings call for reinforcement of tobacco prevention and control policies across Iranian schools and universities.

Keywords: cigarette smoking, adolescents, young adults, prevalence, Islamic Republic of Iran

Citation: Aryaie M; Sharifi H; Khorrami Z; Ardalan G; Rajaei L; Heydari G; et al. Prevalence of cigarette smoking in Iranian adolescents and young adults: a systematic review and meta-analysis. East Mediterr Health J. 2021;27(6):612-628. https://doi.org/10.26719/emhj.21.018

Received: 27/11/19; accepted 23/06/20

Copyright (C) World Health Organization (WHO) 2021. Open Access. Some rights reserved. This work is available under the CC BY-NC-SA 3.0 IGO license (https://creativecommons.org/licenses/by-nc-sa/3.o/igo)
\end{abstract}

\section{Introduction}

Cigarette smoking is one of the most important causes of death and disability worldwide. The World Health Organization (WHO) has estimated that tobacco causes about 8 million deaths annually worldwide (1). Smoking imposes a heavy economic burden globally, especially in developing countries. The diseases attributed to smoking accounted for around 6\% of global health expenditure in 2012 (2). In the WHO Eastern Mediterranean Region, the age-standardized prevalence of smoking in men aged $>15$ years is estimated to be around $40 \%$ (3).

Cigarette smoking usually begins at an early age of adolescence. The early onset of smoking is associated with early incidence of noncommunicable diseases (4) as well as infectious diseases (5). Furthermore, tobacco dependence is more severe in people who start smoking at younger ages, and quitting smoking is more difficult in this group (6-8). The prevalence of adolescent and young adult smoking varies in different regions of the world. In the United States of America (USA), 16\% of young adults aged 18-24 years (9) and $12.5 \%$ of adolescents smoked cigarettes at least once a month (10). A recent estimate indicates that around $10 \%$ of adolescents aged $12-15$ years in low- and middle-income countries smoke cigarettes (11). In the Islamic Republic of Iran, the prevalence of monthly smoking among high school students varies from $2.7 \%$ to $20 \%(12-16)$.

Policy-making requires that we take into account the public health importance of cigarette smoking, and provide timely and comprehensive information on current status of cigarette smoking among adolescents and young adults. In this systematic review, we provided a nationwide estimate of current, regular and life-time cigarette smoking among Iranian adolescents and young adults aged 18-30 years. Furthermore, we reported the gender-specific prevalence of smoking in various subgroups including high school and university students as well as the general population.

\section{Methods}

\section{Study design}

This systematic review was conducted according to the Meta-analysis Of Observational Studies in Epidemiology 
(MOOSE) guidelines. We used the Preferred Reporting Items for Systematic Review and Meta-Analyses (PRISMA) for detailing the identification and selection of studies for inclusion in the review.

\section{Study selection}

Studies were eligible for inclusion if they were cross-sectional; conducted among adolescents aged $14-18$ years or young adults aged 18-30 years; and published in either English or Farsi languages. Studies were excluded if they used nonrandom sampling; the sample size was not clearly declared; the prevalence was not reported or could not be calculated from the data; and the prevalence was reported based on the level of nicotine.

\section{Search strategy}

We searched PubMed, Web of Science, Embase and Scopus to identify English-language articles. To capture Farsi-language articles, databases of SID, Iran Medex, MagIran, Medlib and IranDoc were searched. The search was restricted to January 2001 to December 2018. The searches were adapted for each database, using combinations of the following keywords: (1) prevalence OR epidemiology OR frequency OR cigarette smoking OR occurrence OR incidence OR survey; (2) tobacco smoking OR cigar smoking OR smoking OR smoking behave* OR smoking habit*; and (3) Iran OR Persia*

\section{Article screening}

Two independent reviewers (MA and ZKh) carefully scanned the titles, abstracts and keywords of each article for their relevancy and eligibility. Any disagreements between the reviewers were resolved by further investigation and discussion among the authors. If the information in the title or abstract was insufficient, the full text was reviewed.

\section{Data extraction}

The following information was extracted from each eligible article by 2 independent reviewers, and if there was any discrepancy between the reviewers, the decision was based on negotiation with the third reviewer. The extracted data included: study location and setting, publication year, participants' characteristics (gender and age), sampling source (high school, university, or general population), sample size, reported prevalence of cigarette smoking and its confidence intervals (CIs) by type (lifetime vs current and regular).

\section{Definitions}

Life-time smoking was defined as whether a person had ever smoked cigarettes in their life-time. Cigarette smoking during the previous month was considered as current smoking. Regular smoking was defined as smoking $\geq 1$ cigarette every day or every other day during the previous month. We categorized the prevalence of smoking by level of education, gender, and type of smoking. Furthermore, to increase the generalization of the results, we estimated the prevalence based on the time of the study and type of city. As the prevalence of smoking may vary by time, we estimated the pooled prevalence in 20012010 and 2011-2018. We categorized cities based on their population: small cities with the population $<1$ million and large cities with a population $\geq 1$ million. We calculated the pooled prevalence in subgroups with at least 3 related articles.

\section{Quality assessment}

The quality of studies was examined by two authors (ZKh and MA) based on the STROBE (Strengthening the Reporting of Observational Studies in Epidemiology) checklist for cross-sectional studies.

\section{Statistical analysis}

The pooled prevalence was calculated using the metaprop command in Stata version 14. The standard error of prevalence, $E=\sqrt{\frac{p(1-p)}{n}}$, for each study was calculated based on the binomial distribution formula. The heterogeneity of studies was evaluated using $\mathrm{I}^{2}$ statistics. For $\mathrm{I}^{2}$ $>50 \%$, which depicted heterogeneity between studies, the random effects model was used to estimate the pooled measures and 95\% CIs. A forest plot was used to display the results of the meta-analysis. A funnel plot of log odds versus study size was constructed (17).

\section{Results}

\section{Literature search}

Our search identified 3456 articles, and 1477 of them were excluded because they were duplicates. Title/abstract screening of the remaining 1979 articles resulted in the selection of 475 . Further screening of full-text articles led to the inclusion of 99 eligible articles in the meta-analysis.

Forty-six studies were conducted in high school students and included 51943 girls and 74476 boys. The number of studies on male, female and both genders was 17, 1 and 28, respectively. Twenty-four studies reported current smoking and 33 the lifetime prevalence of cigarette smoking. Daily or regular smoking was reported in 22 studies $(12-14,18-60)$. Thirty-seven studies were conducted among university students; 4 among men and 33 among both genders. There were 37169 female and 25647 male students. Twenty-eight studies reported the current prevalence and 13 the life-time prevalence of smoking. Daily or regular smoking was reported in 10 studies (16,61-96). Finally, 16 studies were conducted in the general population; 1 among women and 15 among both genders. These studies recruited 29676 women and 36587 men. The current and life-time prevalence of smoking was reported in 7 and 1 studies, respectively. Regular smoking was reported in 13 studies (97-112).

\section{Meta-analysis of smolking prevalence}

\section{High school students}

The characteristics of the studies included in the systematic review and meta-analysis of cigarette smoking prevalence in Iranian high school students are presented in 
Table 1. The pooled prevalence ( $95 \% \mathrm{CI})$ of life-time smoking was $13 \%(10-16 \%)$ in girls (Figure $1 \mathrm{~A}$ ) and $23 \%(20-26 \%)$ in boys (Figure 1D). The overall (combining both genders) pooled prevalence of life-time smoking was 18\% (14-23\%) (Figure 2A). The pooled prevalence of current smoking was 3\% (2-4\%) among girls (Figure $1 \mathrm{~B})$ and $9 \%(7-11)$ among boys (Figure 1E). The overall pooled prevalence of current smoking was $7 \%(5-8 \%)$ (Figure $2 \mathrm{~B}$ ). The pooled prevalence of regular smoking was $1 \%(0-1 \%)$ in girls (Figure $1 \mathrm{C}$ ) and $5 \%(4-7 \%)$ in boys (Figure $1 \mathrm{~F}$ ). The overall pooled prevalence of regular smoking was 3\% (2-4) (Figure $2 \mathrm{C}$ ). The pooled prevalence of current smoking in both genders was higher in 2011-2018 than in 2001-2010 ( $4 \%$ vs 3\% among girls and 10\% vs 9\% among boys) (Figure $3 \mathrm{~B}$ and $3 \mathrm{E})$. However, the pooled prevalence of life-time smoking was lower in 2011-2018 than in 2001-2010 (10\% vs $17 \%$ among girls and $23 \%$ vs $25 \%$ among boys) (Figure $3 \mathrm{~A}$ and $3 \mathrm{D}$ ). For regular smoking, there was a decrease in smoking prevalence within the more recent period among girls (0.0\% vs $1 \%)$ but an increase among boys ( $6 \%$ vs $5 \%$ ) (Figure $3 \mathrm{C}$ and $3 \mathrm{~F}$ ). Furthermore, the pooled prevalence of all types of smoking in both genders was higher in large cities than in small cities (Figure 4, see Appendix online).

\section{University students}

The characteristics of the studies included in the systematic review and meta-analysis of cigarette smoking prevalence in Iranian university students are presented in Table 2 . The pooled life-time prevalence of smoking was $11 \%(7-14 \%)$ in women (Figure 5A, see Appendix online) and $33 \%(23-44 \%)$ in men (Figure $5 \mathrm{D}$, see Appendix online). The overall pooled prevalence of life-time smoking was $18 \%(12-24 \%)$ (Figure 2D). The pooled prevalence of current smoking was $5 \%(3-6 \%)$ in women (Figure $5 \mathrm{~B}$, see Appendix online) and 22\% (16-28\%) in men (Figure 5 E, see Appendix online). The overall pooled prevalence of current smoking was $12 \%(10-15 \%)$ (Figure 2E). The pooled prevalence of regular smoking was $1 \%(1-2 \%)$ in women (Figure 5 C, see Appendix online) and $11 \%(7-15 \%)$ in men (Figure 5F, see Appendix online). The overall pooled prevalence of regular smoking was 5\% (3-7\%) (Figure 2F). The pooled prevalence of current smoking in both genders was higher in $2011-2018$ than in $2001-2010$ (2\% vs $1 \%$ among women and $24 \%$ vs $20 \%$ among men) (Figure 6B and 6D, see Appendix online). However, the pooled prevalence of life-time smoking was lower within the more recent period than in the previous years. This increase was greater among men (10\% vs $13 \%$ among women and $29 \%$ vs $49 \%$ among men) (Figure 6A and 6C, see Appendix online). We did not perform the subgroups analysis for regular smoking because of the insufficient number of studies. The pooled prevalence of all types of smoking in both genders was higher in large cities than in small cities (Figure 7, see Appendix online). The only exception was a higher prevalence of current smoking among men in small cities than in large cities (Figure $7 \mathrm{E}$, see Appendix online).

\section{General population}

The studies on smoking prevalence in the general population were heterogeneous (Table 3, see Appendix online); therefore, we could not estimate the pooled prevalence. The prevalence of current smoking among the age group $15-24$ years was reported to be $3.3-13.9 \%$. The prevalence of regular smoking among this age group varied from $1.0 \%$ to $10.2 \%$. The asymmetry in the funnel plots was indicative of some degree of publication bias (Figures 8 and 9, see Appendix online).

\section{Discussion}

The current systematic review and meta-analysis indicated that the prevalence of cigarette smoking was high among Iranian adolescents and young adults. The pattern of smoking differed among different age groups. The prevalence of smoking among male university students was markedly higher than among male high school students, whereas in female students, the difference between the 2 groups was negligible. In both age groups, male students had a higher prevalence of smoking than female students had. Furthermore, the prevalence of smoking was higher in large cities compared to small cities. Around $25 \%$ of Iranian male high school students experienced smoking during their life time. The prevalence of current and regular smoking was $9 \%$ and $5 \%$, respectively, among male high school students. In male university students, a third had experienced life-time smoking; more than $20 \%$ of students were current smokers and $10 \%$ were regular smokers. Around 13\% of female high school students and $11 \%$ of university students had ever smoked cigarettes. The prevalence of current smoking in female university students was only $2 \%$ more than among high school students ( $5 \%$ vs 3\%), and regular smoking in both age groups was similar (1\%).

According to the findings of a nationwide approach to surveillance (STEPS) survey in the Islamic Republic of Iran in 2016, the prevalence of current smoking among adults aged 18-24 years was estimated to be $14 \%$ for men and $2 \%$ for women. In the current study, we showed that among university students, $22 \%$ of men and $5 \%$ of women currently smoked cigarettes. Comparison of our findings with those of the STEPS survey indicate that the prevalence of current smoking among university students is higher than in the general population. Furthermore, a meta-analysis in the Islamic Republic of Iran in 2013 indicated that $19.8 \%$ of male and $2.2 \%$ of female university students smoked cigarettes (113). However, the main drawback of that meta-analysis is the lack of a clear definition for smoking, which limits any comparison. The prevalence of current smoking in young adults varies in different regions of the world. For instance, in Western Cape, South Africa, the prevalence for men and women was $22 \%$ and $14.8 \%$, respectively (114). According to the 2013 National Health Interview Survey in the USA, the prevalence of current cigarette smoking in men and women aged 18-25 years was approximately $22 \%$ and $15 \%$, respectively (115). Furthermore, in the 
Table 1 Characteristics of studies included in systematic review and meta-analysis of cigarette smoking prevalence in Iranian high school students categorized by type of use

\begin{tabular}{|c|c|c|c|c|c|c|c|c|}
\hline \multirow[t]{2}{*}{ Author } & \multirow[t]{2}{*}{$\begin{array}{c}\text { Year of } \\
\text { publication }\end{array}$} & \multirow[t]{2}{*}{ City } & \multicolumn{3}{|c|}{ Sample size } & \multicolumn{3}{|c|}{$\begin{array}{c}\text { Current smoking prevalence } \\
(\%)\end{array}$} \\
\hline & & & Total & Female & Male & Total & Female & Male \\
\hline Ziaee & 2001 & Tehran & 4023 & 2018 & 2005 & 4.1 & 1.0 & 7.2 \\
\hline Ahmadi & 2003 & Shiraz & 397 & 200 & 197 & 8.3 & 1.5 & 15.2 \\
\hline Kelishadi & 2004 & Isfahan, Najaf Abad, Arak & 1950 & 1004 & 946 & 8.7 & 4.0 & 12.5 \\
\hline Mojahed & 2004 & Zahedan & 475 & 259 & 216 & 1.3 & 0.4 & 2.3 \\
\hline Vafaee & 2004 & Tabriz & 1000 & - & - & 28.6 & - & - \\
\hline Yazdani & 2008 & Tehran & 502 & 242 & 260 & 3.5 & 2.0 & 5.0 \\
\hline Namkin & 2008 & Birjand & - & - & 1233 & - & - & 3.9 \\
\hline Ramezankhani & 2010 & Tehran & 2340 & 1079 & 1216 & 11.4 & 10.1 & 13.1 \\
\hline Rahmanian & 2010 & Jahrom & 1145 & 456 & 689 & 9.9 & 3.3 & 14.2 \\
\hline Mohammadkhani & 2010 & 10 provinces & 2538 & 1255 & 1283 & 4.4 & 1.3 & 7.4 \\
\hline Karimy & 2012 & Zarand & - & - & 250 & - & - & 14.7 \\
\hline Habib E & 2012 & Tehran & 4591 & 2499 & 2092 & 8.3 & 5.3 & 12.1 \\
\hline Moeini & 2012 & Hamedan & 1161 & 573 & 588 & 10.2 & 6.8 & 13.4 \\
\hline Shahnazi & 2012 & Isfahan & - & - & 382 & - & - & 7.2 \\
\hline Mohammadi & 2014 & Babolsar & - & - & 450 & - & - & 7.4 \\
\hline Karimy & 2014 & Zarandieh & - & - & 350 & - & - & 15.1 \\
\hline Reisi & 2014 & Isfahan & - & - & 382 & - & - & 32.0 \\
\hline Meysamie & 2015 & Tehran & 2877 & 1320 & 1557 & 4.4 & 1.4 & 7.0 \\
\hline Alizadeh-Charandabi & 2015 & Sanandaj & 1524 & 760 & 764 & 9.9 & 6.4 & 13.1 \\
\hline Roohafza & 2015 & Isfahan & 5362 & 2641 & 2681 & 5.8 & 1.9 & 9.7 \\
\hline Mohammadi & 2017 & Marivan & - & - & 470 & - & - & 6.4 \\
\hline Rezaei & 2017 & Jahrom & 630 & 315 & 315 & 2.7 & 1.6 & 3.8 \\
\hline Bashirian & 2018 & Hamedan & - & - & 730 & - & - & 13.2 \\
\hline Sabzmakan & 2018 & Noshahr & - & - & 440 & - & - & 11.8 \\
\hline \multirow[t]{2}{*}{ Author } & \multirow{2}{*}{$\begin{array}{c}\text { Year of } \\
\text { publication }\end{array}$} & \multirow[t]{2}{*}{ City } & \multicolumn{3}{|c|}{ Sample size } & \multicolumn{3}{|c|}{ Lifetime prevalence } \\
\hline & & & Total & Female & Male & Total & Female & Male \\
\hline Ziaee & 2001 & Tehran & 4023 & 2018 & 2005 & 30.9 & 26.9 & 35.0 \\
\hline Ahmadi & 2003 & Shiraz & 397 & 200 & 197 & 25.4 & 11.5 & 39.5 \\
\hline Mojahed & 2004 & Zahedan & 475 & 259 & 216 & 15.6 & 7.8 & 25.2 \\
\hline Mojahed & 2004 & Zahedan & 475 & 259 & 216 & 1.6 & 1.6 & 0.8 \\
\hline Mohammadpoorasl & 2007 & Tabriz & - & - & 1779 & - & - & 18.2 \\
\hline Mohtasham Amiri & 2008 & Rasht & - & - & 1297 & - & - & 28.2 \\
\hline Namkin & 2008 & Birjand & - & - & 1233 & - & - & 21.3 \\
\hline Pasharosh & 2009 & Kermanshah & - & 3163 & - & - & 15.0 & - \\
\hline Rahmanian & 2010 & Jahrom & 1145 & - & - & 21.9 & - & - \\
\hline Ramezankhani & 2010 & Tehran & 2340 & 1079 & 1216 & 36.3 & 36.5 & 37.6 \\
\hline Momtazi & 2010 & Zanjan & 537 & 273 & 264 & 21.8 & 10.3 & 33.7 \\
\hline Mohammadkhani & 2010 & 10 province & 2538 & 1255 & 1283 & 14.7 & 6.1 & 23.1 \\
\hline Mohammadpoorasl & 2011 & Tabriz & - & - & 1785 & - & - & 14.3 \\
\hline Shamshiri Milani & 2011 & Tehran & - & 2313 & - & - & 12.9 & - \\
\hline Mohammadkhani & 2011 & National & 2538 & 1255 & 1238 & 14.7 & 6.1 & 23.1 \\
\hline Karimy & 2012 & Zarand & - & - & 250 & - & - & 43.7 \\
\hline Mohammadpoorasl & 2012 & Tabriz & 4903 & 2775 & 2099 & 16.4 & 11.2 & 22.5 \\
\hline Shahnazi & 2012 & Isfahan & - & - & 382 & - & - & 32.7 \\
\hline Nazarzadeh & 2013 & Zanjan & - & - & 1064 & - & - & 23.4 \\
\hline Pirdehghan & 2013 & Yazd & 460 & 187 & 273 & 23.3 & 12.8 & 30.8 \\
\hline Mohammadi & 2014 & Babolsar & - & - & 450 & - & - & 30.1 \\
\hline Barati & 2014 & Hamedan & - & - & 810 & - & - & 17.2 \\
\hline
\end{tabular}


Table 1 Characteristics of studies included in systematic review and meta-analysis of cigarette smoking prevalence in Iranian high school students categorized by type of use (concluded)

\begin{tabular}{|c|c|c|c|c|c|c|c|c|}
\hline \multirow[t]{2}{*}{ Author } & \multirow{2}{*}{$\begin{array}{c}\text { Year of } \\
\text { publication }\end{array}$} & \multirow[t]{2}{*}{ City } & \multicolumn{3}{|c|}{ Sample size } & \multicolumn{3}{|c|}{ Lifetime prevalence } \\
\hline & & & Total & Female & Male & Total & Female & Male \\
\hline Khajehdaluee & 2014 & Sarakhs & 943 & 436 & 507 & 19.2 & 13.8 & 23.9 \\
\hline Bidel & 2014 & Ilam & - & - & 1000 & - & - & 11.4 \\
\hline Meysamie & 2015 & Tehran & 2877 & 1320 & 1557 & 12.1 & 9.9 & 13.8 \\
\hline Roohafza & 2015 & Isfahan & 5362 & 2641 & 2681 & 11.6 & 6.8 & 16.5 \\
\hline Chaman & 2015 & Shahroud & - & - & 450 & - & - & 12.8 \\
\hline Ayubi & 2017 & Zanjan & - & - & 958 & - & - & 23.4 \\
\hline Karimi & 2017 & Shiraz & - & - & 900 & - & - & 19.7 \\
\hline Khayyati & 2017 & East Azerbaijan & 4422 & 1990 & 2432 & 8.9 & $5 \cdot 5$ & 11.8 \\
\hline Mohammadi & 2017 & Marivan & - & - & 470 & - & - & 34.7 \\
\hline Pirdehghan & 2017 & Yazd & 704 & 256 & 448 & 17.4 & 9.8 & 21.9 \\
\hline Bashirian & 2018 & Hamedan & - & - & 730 & - & - & 27.2 \\
\hline \multirow[t]{2}{*}{ Author } & \multirow{2}{*}{$\begin{array}{c}\text { Year of } \\
\text { publication }\end{array}$} & \multirow[t]{2}{*}{ City } & \multicolumn{3}{|c|}{ Sample size } & \multicolumn{3}{|c|}{ Regular } \\
\hline & & & Total & Female & Male & Total & Female & Male \\
\hline Heidari & 2004 & Tehran & 1119 & - & - & 3.4 & - & - \\
\hline Mojahed & 2004 & Zahedan & 475 & 259 & 216 & 1.2 & 0.4 & 2.3 \\
\hline Heydari & 2007 & Tehran & 1095 & 381 & 712 & 5.0 & 2.0 & 6.0 \\
\hline Mohammadpoorasl & 2007 & Tabriz & - & - & 1779 & - & - & 4.4 \\
\hline Mohtasham Amiri & 2008 & Rasht & - & - & 1297 & - & - & 6.7 \\
\hline Pasharosh & 2009 & Kermanshah & - & 3163 & - & - & 1.1 & - \\
\hline Rahmanian & 2010 & Jahrom & 1145 & - & - & 14.2 & - & - \\
\hline Ramezankhani & 2010 & Tehran & 2340 & 1079 & 1216 & $3 \cdot 3$ & 2.1 & 4.2 \\
\hline \multirow[t]{2}{*}{ Author } & \multirow{2}{*}{$\begin{array}{c}\text { Year of } \\
\text { publication }\end{array}$} & \multirow[t]{2}{*}{ City } & \multicolumn{3}{|c|}{ Sample size } & \multicolumn{3}{|c|}{ Regular } \\
\hline & & & Total & Female & Male & Total & Female & Male \\
\hline Mohammadpoorasl & 2011 & Tabriz & - & - & 1785 & - & - & 2.8 \\
\hline Mohammadpoorasl & 2012 & Tabriz & 4903 & 2775 & 2099 & 4.0 & 0.5 & 9.2 \\
\hline Nazarzadeh & 2013 & Zanjan & - & - & 1064 & - & - & 10.8 \\
\hline Heydari & 2013 & Tehran & - & - & 1271 & - & - & 6.3 \\
\hline Bidel & 2014 & Ilam & - & - & 1000 & - & - & 1.3 \\
\hline Mohammadi & 2014 & Babolsar & - & - & 450 & - & - & $7 \cdot 5$ \\
\hline Mohammadi & 2014 & Babolsar & - & - & 450 & - & - & 2.3 \\
\hline Barati & 2014 & Hamedan & & & 810 & & & 2.7 \\
\hline Meysamie & 2015 & Tehran & 2877 & 1320 & 1557 & 2.4 & 0.2 & 4.3 \\
\hline Mohammadi & 2017 & Marivan & - & - & 470 & - & - & 4.7 \\
\hline Khayyati & 2017 & East Azerbaijan & 4422 & 1990 & 2432 & 2.0 & 0.6 & 3.1 \\
\hline Ayubi & 2017 & Zanjan & - & - & 958 & - & - & 10.8 \\
\hline Ataeias & 2018 & Tabriz & 1161 & 566 & 567 & 5.9 & 1.2 & 10.6 \\
\hline
\end{tabular}

Eastern Mediterranean Region, the prevalence of current cigarette smoking in university students was $11 \%$ in Pakistan (116), $18 \%$ in Palestine (32.8\% in men vs $3.6 \%$ in women) (117) and $24.7 \%$ in the Syrian Arab Republic (39.82\% in men vs $5.54 \%$ in women) (118). The global estimates in health profession students showed that the prevalence of smoking varies by region. The highest prevalence was reported in Europe, where the prevalence in men ranged from $36 \%$ among nursing students to $55.8 \%$ among pharmacy students. In the Eastern Mediterranean Region, the prevalence varied from $3 \%$ to $6 \%$ in women and $20 \%$ to $28 \%$ in men (119). Compared with these national reports, in our study, the prevalence of smoking among Iranian students, especially women, seems to be lower than in European countries, but similar to most Eastern Mediterranean countries. One explanation for the lower prevalence of cigarette smoking in the Eastern Mediterranean Region, including the Islamic Republic of Iran, may be the increasing tendency of young adults to try other types of tobacco, mainly water pipe smoking. In the Islamic Republic of Iran, the prevalence of life-time and current water-pipe smoking among students was 
Figure 1 Forest plot of studies included in meta-analysis of cigarette smoking prevalence in high school students: Left: life time (A), current (B) and regular (C) smoking prevalence in girls. Right: life time (D), current (E), regular (F) smoking prevalence in boys.
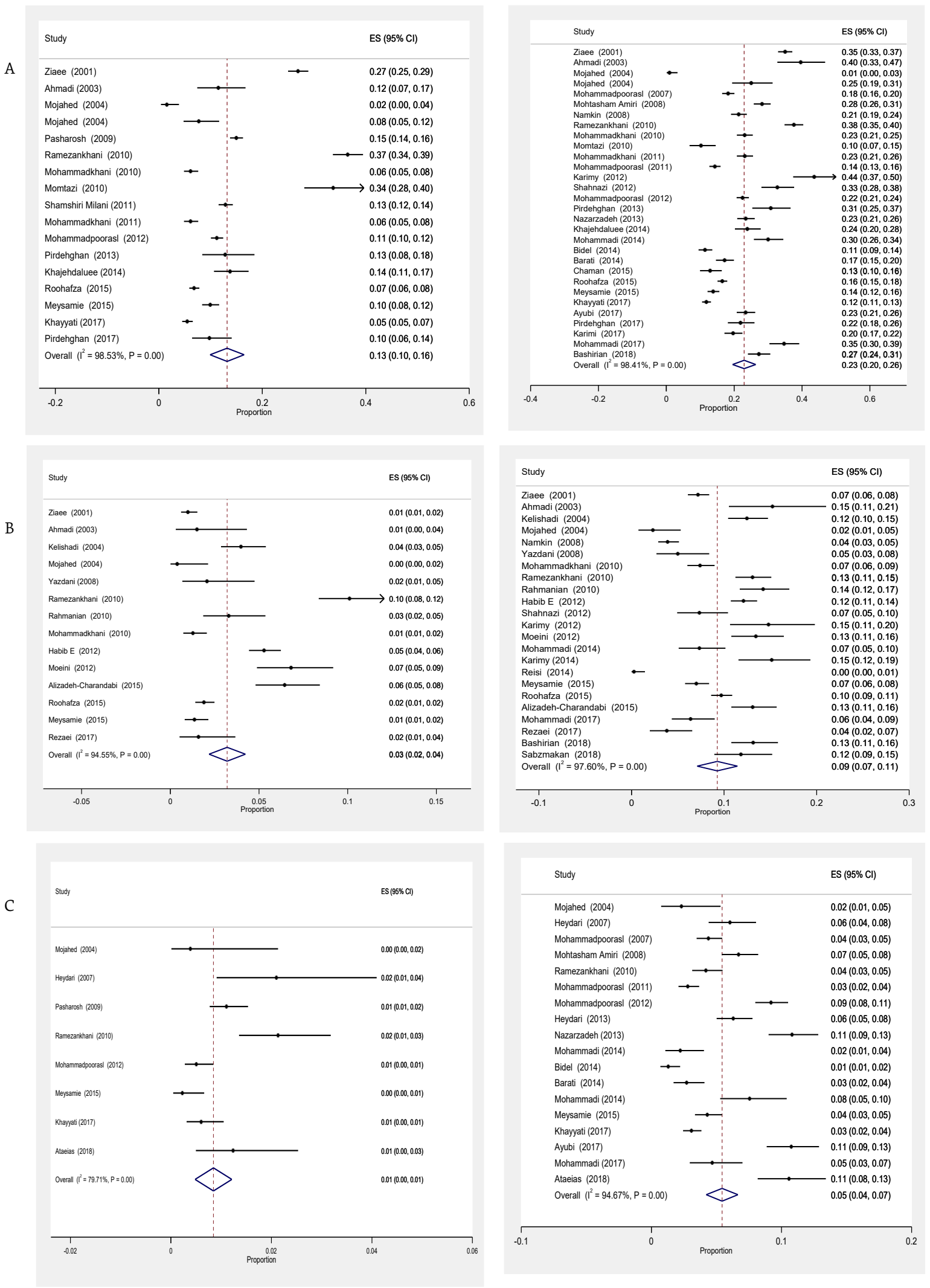
Figure 2 Forest plot of studies included in meta-analysis of total (both sexes) cigarette smoking prevalence in high school and university students. Left: life time (A), current (B) and regular (C) smoking prevalence in high school students. Right: life time (D), current (E) and regular (F) smoking prevalence in university students.
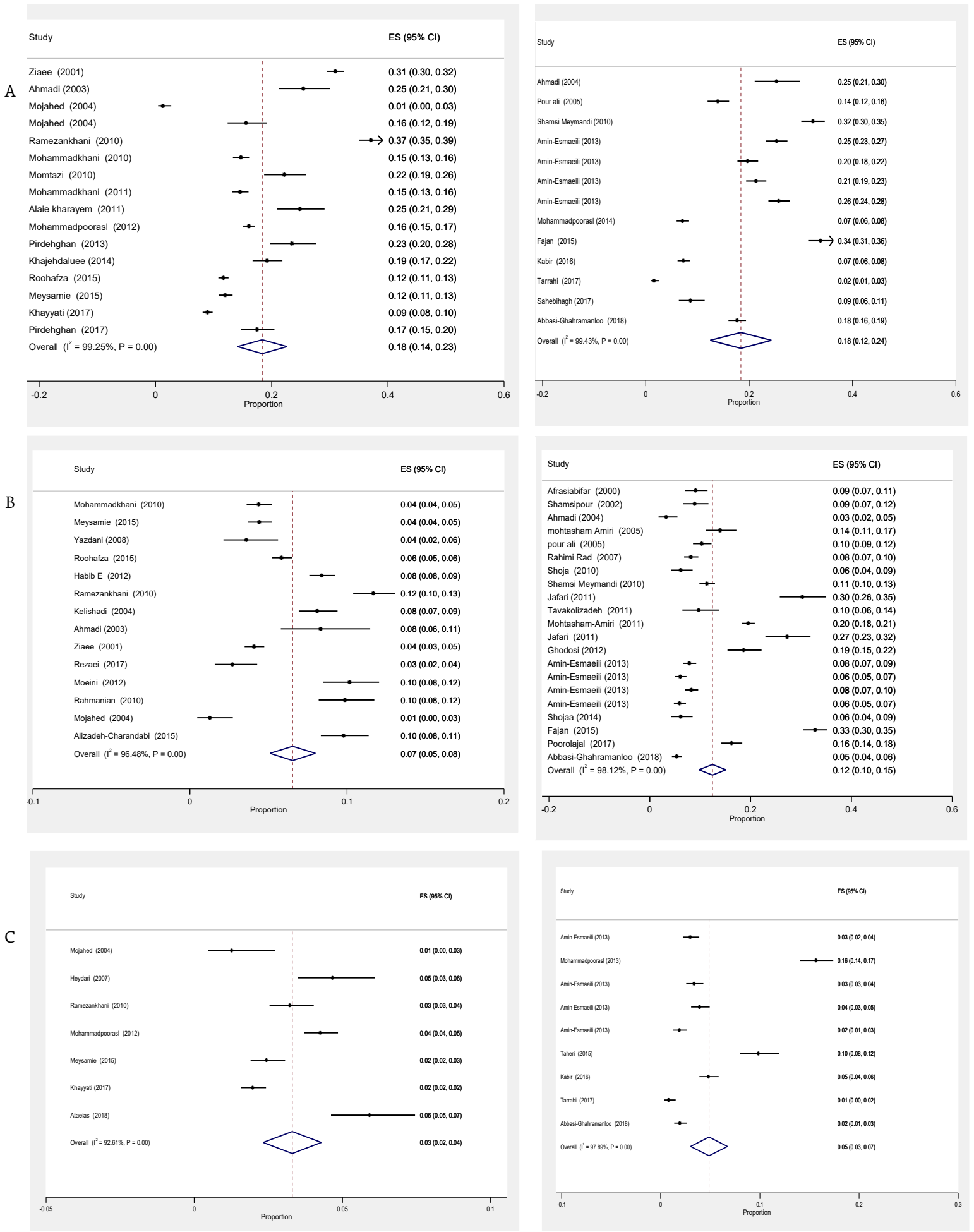


\begin{tabular}{|c|c|c|c|c|c|c|c|c|}
\hline \multirow[t]{2}{*}{ Author } & \multirow{2}{*}{$\begin{array}{c}\text { Year of } \\
\text { publication }\end{array}$} & \multirow[t]{2}{*}{ City } & \multicolumn{3}{|c|}{ Sample size } & \multicolumn{3}{|c|}{ Life-time prevalence } \\
\hline & & & Total & Female & Male & Total & Female & Male \\
\hline Ahmadi & 2004 & Shiraz & 400 & 343 & 57 & 25.3 & 19.4 & 59.3 \\
\hline Pour ali & 2005 & Ardebil & 1106 & 779 & 327 & 13.9 & 3.7 & 38.3 \\
\hline Zarrabi & $2005-2006$ & Gilun & 827 & - & - & 26.4 & - & - \\
\hline Shamsi Meymandi & 2010 & Kerman & 1677 & 872 & 805 & 31.0 & 15.0 & 51.0 \\
\hline Amin-Esmaeili & 2013 & Tehran & 1761 & 1162 & 599 & 34.0 & 16.4 & 43.8 \\
\hline Amin-Esmaeili & 2013 & Tehran & 1741 & 1107 & 629 & 32.7 & 15.4 & 42.8 \\
\hline Amin-Esmaeili & 2013 & Tehran & 1755 & 1159 & 591 & 29.6 & 12.5 & 38.5 \\
\hline Amin-Esmaeili & 2013 & Tehran & 1568 & 1043 & 518 & 31.7 & 10.0 & 39.1 \\
\hline Mohammadpoorasl & 2014 & Tabriz & 1837 & 1100 & 737 & 15.6 & 4.2 & 11.4 \\
\hline Fajani & 2015 & Isfahan & 1512 & 765 & 747 & 33.7 & 22.8 & 44.9 \\
\hline Jalilian & 2016 & Kermanshah & 620 & - & - & 11.6 & - & - \\
\hline Kabir & 2016 & Karaj & 1959 & 1301 & 658 & 7.2 & 6.3 & 9.1 \\
\hline Mozafarinia & 2017 & Tehran & 422 & - & - & 26.3 & - & - \\
\hline Sahebihagh & 2017 & Qazvin & 521 & 357 & 164 & 8.6 & 3.0 & 20.7 \\
\hline Tarrahi & 2017 & Khorramabad & 1131 & 701 & 430 & 1.6 & 0.9 & 2.8 \\
\hline Abbasi-Ghahramanloo & 2018 & Tehran & 1985 & 1376 & 609 & 17.6 & 10.6 & 33.5 \\
\hline \multirow[t]{2}{*}{ Author } & \multirow{2}{*}{$\begin{array}{c}\text { Year of } \\
\text { publication }\end{array}$} & \multirow[t]{2}{*}{ City } & \multicolumn{3}{|c|}{ Sample size } & \multicolumn{3}{|c|}{ Regular prevalence } \\
\hline & & & Total & Female & Male & Total & Female & Male \\
\hline Nakhaee & 2009 & Kerman & 833 & - & - & 4.5 & - & - \\
\hline Mohtasham-Amiri & 2011 & Guilan & 3700 & - & - & 16.0 & - & - \\
\hline Amin-Esmaeili & 2013 & Tehran & 1761 & 1162 & 599 & 5.9 & 0.6 & 8.7 \\
\hline Amin-Esmaeili & 2013 & Tehran & 1741 & 1107 & 629 & 6.2 & 0.9 & 9.3 \\
\hline Amin-Esmaeili & 2013 & Tehran & 1755 & 1159 & 591 & $5 \cdot 3$ & 0.4 & 7.9 \\
\hline Amin-Esmaeili & 2013 & Tehran & 1568 & 1043 & 518 & 3.9 & 0.1 & 5.4 \\
\hline Mohammadpoorasl & 2013 & Tabriz & 1837 & 1100 & 734 & 15.8 & 7.1 & 28.5 \\
\hline Taheri & 2015 & Mashhad & 936 & 519 & 417 & 9.8 & 2.5 & 18.9 \\
\hline Kabir & 2016 & Karaj & 1959 & 1301 & 658 & 4.8 & 1.2 & 11.9 \\
\hline Menati & 2016 & Ilam & & & 1824 & & & 16.6 \\
\hline Mozafarinia & 2017 & Tehran & 422 & - & - & 8.5 & - & - \\
\hline Tarrahi & 2017 & Khorramabad & 1131 & 701 & 430 & 0.8 & 0.3 & 1.6 \\
\hline Abbasi-Ghahramanloo & 2018 & Tehran & 1985 & 1376 & 609 & 1.9 & 0.2 & 5.7 \\
\hline \multirow[t]{2}{*}{ Author } & \multirow{2}{*}{$\begin{array}{c}\text { Year of } \\
\text { publication }\end{array}$} & \multirow[t]{2}{*}{ City } & \multicolumn{3}{|c|}{ Sample size } & \multicolumn{3}{|c|}{ Current prevalence } \\
\hline & & & Total & Female & Male & Total & Female & Male \\
\hline Ahmadi & 2004 & Shiraz & 400 & 343 & 57 & 3.3 & 1.2 & 15.3 \\
\hline pour ali & 2005 & Ardebil & 1106 & 779 & 327 & 7.4 & 1.2 & 32.0 \\
\hline Zarrabi & $2005-2006$ & Guilan & 827 & - & - & 9.6 & - & - \\
\hline Nojomi & 2006 & Tehran & 1000 & - & - & 5.6 & - & - \\
\hline Rahimi Rad & 2007 & Urmia & 1500 & 908 & 592 & 8.1 & 2.3 & 17.1 \\
\hline Mohtasham-Amiri & 2009 & Astara & 1226 & - & - & 19.5 & - & - \\
\hline Shoja & 2010 & Gorgan & 538 & 362 & 176 & 6.1 & 0.8 & 17.3 \\
\hline Shamsi Meymandi & 2010 & Kerman & 1677 & 872 & 805 & 11.0 & 2.0 & 21.5 \\
\hline Nazary & 2010 & Semnan & - & - & 320 & - & - & 14.4 \\
\hline Kasiri & 2011 & Ahvaz & - & - & 745 & - & - & 15.0 \\
\hline Jafari & 2011 & Tehran & 400 & 201 & 199 & 30.0 & 15.9 & 44.7 \\
\hline Tavakolizadeh & 2011 & Gonabad & 279 & 125 & 154 & 9.8 & 4.1 & 14.4 \\
\hline Jafari & 2011 & Tehran & 400 & 143 & 257 & 27.3 & 12.6 & 35.4 \\
\hline Mohtasham-Amiri & 2011 & Guilan & 3700 & 1870 & 1800 & 19.5 & 6.5 & 33.0 \\
\hline
\end{tabular}




\begin{tabular}{|c|c|c|c|c|c|c|c|c|}
\hline \multirow[t]{2}{*}{ Author } & \multirow{2}{*}{$\begin{array}{c}\text { Year of } \\
\text { publication }\end{array}$} & \multirow[t]{2}{*}{ City } & \multicolumn{3}{|c|}{ Sample size } & \multicolumn{3}{|c|}{ Current prevalence } \\
\hline & & & Total & Female & Male & Total & Female & Male \\
\hline Ghodsi & 2012 & Guilan & - & - & 222 & - & - & 23.0 \\
\hline Ghodosi & 2012 & Isfahan & 537 & 292 & 245 & 18.6 & 4.5 & 35.5 \\
\hline Jamali & 2013 & Tehran & 1086 & - & - & 11.9 & - & - \\
\hline Amin-Esmaeili & 2013 & Tehran & 1761 & 1162 & 599 & 12.4 & 3.8 & 16.8 \\
\hline Amin-Esmaeili & 2013 & Tehran & 1741 & 1107 & 629 & 11.9 & 2.4 & 17.4 \\
\hline Amin-Esmaeili & 2013 & Tehran & 1755 & 1159 & 591 & 11.2 & 2.0 & 14.1 \\
\hline Amin-Esmaeili & 2013 & Tehran & 1568 & 1043 & 518 & 10.5 & 1.3 & 15.1 \\
\hline Machowicz & 2013 & Tehran & 170 & - & - & 3.5 & - & - \\
\hline Nasirian & 2013 & Kerman & 772 & - & - & 15.8 & - & - \\
\hline Seghatoleslam & 2014 & Tehran & 500 & - & - & 27.0 & - & - \\
\hline Shojaa & 2014 & Gorgan & 538 & 362 & 176 & 6.1 & 0.8 & 17.0 \\
\hline Babaei Heydarabadi & 2015 & Tehran & 604 & - & - & 10.4 & - & - \\
\hline Fajani & 2015 & Isfahan & 1512 & 765 & 747 & 32.8 & 27.4 & 38.2 \\
\hline Mozafarinia & 2017 & Tehran & 422 & - & - & 3.8 & - & - \\
\hline Poorolajal & 2017 & Hamedan & 1259 & 765 & 494 & 16.2 & 6.3 & 31.6 \\
\hline Abbasi-Ghahramanloo & 2018 & Tehran & 1985 & 1376 & 609 & $5 \cdot 3$ & 1.5 & 14.0 \\
\hline Rahimi Pordanjani & 2018 & Yazd & 250 & - & - & 19.2 & - & - \\
\hline
\end{tabular}

$42 \%$ and $19 \%$, respectively (120). Lack of awareness of the negative effects of water-pipe smoking and lower stigma toward its use are factors that explain this pattern (121).

In male high school students, the prevalence of current cigarette smoking was lower than among university students ( $9 \%$ vs $22 \%$ ). However, for girls and women, the prevalence was similar among high school and university students ( $3 \%$ vs $5 \%$ ). A meta-analysis of $12-15$-year-old adolescents of 68 low- and middle-income countries indicated that $13.3 \%$ of boys and $6 \%$ of girls currently smoked cigarettes (11). The highest prevalence for boys was for the Western Pacific Region (17.5\%) followed by the Eastern Mediterranean Region (13.3\%), whereas for girls, the highest prevalence was reported in the Western Pacific Region (10.0\%) and Region of the Americas (8.7\%). The corresponding prevalence in the Eastern Mediterranean Region for girls was estimated at 3.8\%, which was close to our estimate (11), while the prevalence of current smoking among Iranian high school boys (9\%) seems to be lower than the overall estimate in the Eastern Mediterranean Region (13.3\%).

We showed that in both age groups, male students smoked cigarettes more than female students smoked. The sex ratio varied from 2 (for life-time smoking in high school) to 11 (for regular smoking among university students). Global estimates indicate that men smoke 5 times as much as women smoke (124). A variety of national studies have confirmed the higher prevalence of smoking in Iranian men $(122,123)$. The lower prevalence of cigarette smoking among women may be due to greater stigma that exists toward smoking among women.
We found that the prevalence of smoking was higher in large cities compared to small cities. Despite a slight increase in prevalence of smoking within more recent years, the prevalence of life-time smoking decreased. This decrease may have been due to several factors including increasing awareness of young people about tobacco hazards, tendency to use other drugs, including hookah, and success of tobacco control programmes. In the Islamic Republic of Iran, it is culturally inappropriate for girls to smoke (124). Around $15 \%$ of the 80 million Iranian population is aged 15-24 years and half of them are men. Among $>2$ million high school students and $\sim 4$ million university students, more than half are male $(125,126)$. Extrapolating our results to these populations reflects the alarming rate of cigarette smoking among Iranian adolescents. A growing body of evidence indicates that smoking initiation at lower ages is strongly related to subsequent smoking behaviour, including regular and heavy smoking in adulthood, which highlights the importance of early interventions for smoking prevention (127). According to the World Health Organization report on the national tobacco control programme, the Islamic Republic of Iran has performed well compared to other countries in the Eastern Mediterranean Region in many aspects of smoking control (including monitoring and smoke-free policies, smoking cessation programmes, health warnings on cigarette packages, and mass media and advertising bans for tobacco use). However, more action is needed in the field of taxation and affordability of tobacco, especially in large cities $(128,129)$. The nature of the tobacco epidemic requires a comprehensive prevention and intervention plan focusing on the community as well as schools. A variety of strategies should be reinforced 


\begin{tabular}{|c|c|c|c|c|c|c|c|c|c|}
\hline \multicolumn{10}{|c|}{$\begin{array}{l}\text { Table } 3 \text { Characteristics of studies included in systematic review of cigarette sm } \\
14-30 \text { years }\end{array}$} \\
\hline \multirow[t]{2}{*}{ Author } & \multirow{2}{*}{$\begin{array}{c}\text { Year of } \\
\text { publication }\end{array}$} & \multirow[t]{2}{*}{ City } & \multirow{2}{*}{$\begin{array}{c}\text { Age } \\
\text { group, } \\
\text { yr }\end{array}$} & \multicolumn{3}{|c|}{ Sample size } & \multicolumn{3}{|c|}{ Current prevalence } \\
\hline & & & & Total & Female & Male & Total & Female & Male \\
\hline Mosavi-Jarrahi & 2004 & National & $(15-24)$ & 7984 & 5016 & 2968 & 5.5 & 0.7 & 13.5 \\
\hline Sadeghi & 2004 & Isfahan, Najafabad and Arak & $(26-35)$ & 3786 & 1969 & 1817 & 18.5 & 2.1 & 36.5 \\
\hline Sadeghi & 2004 & $\begin{array}{l}\text { Isfahan Province (Isfahan, } \\
\text { Najafabad and Arak) }\end{array}$ & $(19-25)$ & 3786 & 1969 & 1817 & 11.8 & 0.5 & 24.2 \\
\hline Mosavi-Jarrahi & 2004 & National & $(15-24)$ & 12060 & 6761 & 5299 & 3.3 & 0.3 & 7.2 \\
\hline Fotouhi & 2009 & Tehran & $(15-24)$ & 1043 & 654 & 389 & 3.4 & 0.9 & 6.1 \\
\hline Fotouhi & 2009 & Tehran & $(15-24)$ & 618 & 384 & 234 & 13.9 & 3.2 & 23 \\
\hline Nadimi & 2009 & Rafsanjan & $(20-29)$ & 140 & 71 & 69 & 19.3 & 8.5 & 30.4 \\
\hline Baheiraei & 2013 & Tehran & $(15-18)$ & 1201 & 609 & 592 & 11.4 & 7.2 & 15.7 \\
\hline Abdollahifard & 2013 & Shiraz & $(18-36)$ & 483 & - & - & 5.2 & - & - \\
\hline Shuja & 2017 & Isfahan Province & $(25-34)$ & 2902 & - & - & 15.8 & - & - \\
\hline Shuja & 2017 & Isfahan Province & $(19-24)$ & 1874 & - & - & 11.5 & - & - \\
\hline \multirow[t]{2}{*}{ Author } & \multirow{2}{*}{$\begin{array}{l}\text { Year of } \\
\text { publication }\end{array}$} & \multirow[t]{2}{*}{ City } & & \multicolumn{3}{|c|}{ Sample size } & \multicolumn{3}{|c|}{ Life-time prevalence } \\
\hline & & & $\begin{array}{l}\text { group, } \\
\text { yr }\end{array}$ & Total & Female & Male & Total & Female & Male \\
\hline Baheiraei & 2013 & Tehran & $(15-18)$ & 1201 & 609 & 592 & 26.2 & 22.2 & 30.2 \\
\hline \multirow[t]{2}{*}{ Author } & \multirow{2}{*}{$\begin{array}{l}\text { Year of } \\
\text { publication }\end{array}$} & \multirow[t]{2}{*}{ City } & \multirow{2}{*}{$\begin{array}{l}\text { Age } \\
\text { group }\end{array}$} & \multicolumn{3}{|c|}{ Sample size } & \multicolumn{3}{|c|}{ Regular prevalence } \\
\hline & & & & Total & Female & Male & Total & Female & Male \\
\hline Mehrabi & 2007 & National & $(15-24)$ & 16948 & 8330 & 8618 & 3.7 & 0.7 & 6.8 \\
\hline Mehrabi & 2007 & National & $(25-34)$ & 16934 & 8380 & 8554 & 14.0 & 2.5 & 25.3 \\
\hline Yunesian & 2008 & Tehran & $(13-19)$ & 4249 & 2614 & 1635 & 0.9 & 0.4 & 1.7 \\
\hline Moghimbeigi & 2009 & $\begin{array}{l}\text { Northwest provinces of } \\
\text { Iran }\end{array}$ & $(15-20)$ & 1745 & 935 & 809 & 10.2 & 4.9 & 12.3 \\
\hline Meysamie & 2010 & National & $(25-34)$ & 12760 & - & - & 11.3 & - & - \\
\hline Meysamie & 2010 & National & $(15-24)$ & 17710 & - & - & 5.8 & - & - \\
\hline Baheiraei & 2013 & Tehran & $(15-18)$ & 1201 & 609 & 592 & 2.5 & 0.4 & 4.5 \\
\hline Baheiraei & 2013 & Tehran & $(15-18)$ & 1201 & 609 & 592 & 3.16 & 0.82 & 5.5 \\
\hline Nekoei Moghadam & 2013 & Hormozgan & $(15-24)$ & 200 & 100 & 100 & 1.0 & 0.0 & 2.0 \\
\hline Nekoei Moghadam & 2013 & Hormozgan & $(25-34)$ & 200 & 100 & 100 & 8.5 & 0.0 & 17.0 \\
\hline Baheiraei & 2014 & Tehran & $\begin{array}{l}\text { Female } \\
(25-34)\end{array}$ & - & 468 & - & - & 2.8 & - \\
\hline Baheiraei & 2014 & Tehran & $\begin{array}{l}\text { Female } \\
(15-24)\end{array}$ & - & 350 & - & - & 2.6 & - \\
\hline Hassanzadeh & 2015 & Tehran & $(20-30)$ & 7350 & - & - & 3.7 & - & - \\
\hline Salimzadeh & 2016 & Kerman & $(15-19)$ & 1000 & 500 & 500 & 0.3 & 0.0 & 0.5 \\
\hline Salimzadeh & 2016 & Kerman & $(20-24)$ & 1000 & 500 & 500 & 2.8 & 0.0 & 5.4 \\
\hline Hoseini & 2016 & Tehran & $(15-24)$ & 39 & - & - & 7.7 & - & - \\
\hline Salimzadeh & 2016 & Kerman & $(20-24)$ & 1000 & 500 & 500 & 2.0 & 0.7 & 3.3 \\
\hline Kassani & 2016 & Tehran & $(20-30)$ & - & 14347 & 6.21 & - & - & - \\
\hline Salimzadeh & 2016 & Kerman & $(25-34)$ & 1000 & 500 & 500 & 8.9 & 0.1 & 16.5 \\
\hline Salimzadeh & 2016 & Kerman & $(25-34)$ & 1000 & 500 & 500 & 2.3 & 0.3 & 4.2 \\
\hline
\end{tabular}


to reduce the prevalence and health effects of cigarette smoking in adolescents. Existing laws to restrict access to tobacco products by banning sales to minors should be reinforced. Effective and comprehensive educational programmes on the health risks of tobacco consumption and exposure to tobacco smoke should be established in schools and universities. Additionally, tobacco cessation facilities should be extended to all areas. Involvement of nongovernmental organizations and parents in tobacco control programmes as well as licensing the distribution of tobacco products in order to decrease availability are other strategies that should be strengthened by policymakers.

Our findings should be interpreted by taking into consideration the limitations of the study. There were high variability and heterogeneity among studies regarding the sampling method, study instruments and reporting of variables. For example, despite the use of random sampling in all recruited studies, the variations in the sampling approach among studies were indicative of possible selection bias. Similarly, the different instruments used in the studies were suggestive of some degree of information bias. While definitions regarding life-time smoking were consistent among the included studies, there was some degree of heterogeneity regarding the definition of current and regular smoking. For example, in most studies, an exact definition of current smoking was not used, but in a small number of studies, it was measured objectively. Therefore, our results should be interpreted with caution. Furthermore, due to the lack of comprehensive databases, we did not have access to grey literature. Nevertheless, this review is important as it provides a comprehensive picture regarding the prevalence of smoking among Iranian young people that could serve as the foundation for policy-makers to develop and implement effective smoking prevention and intervention programmes.

\section{Conclusion}

Cigarette smoking is notable among Iranian young adults in a way that boys and university students are affected more. Prevention programmes in high schools and universities should focus on information, education and communication, and current policies on tobacco control should be reinforced.

Funding: None.

Competing interests: None declared.

\section{Prévalence du tabagisme par cigarettes chez les adolescents et les jeunes adultes iraniens : revue systématique et méta-analyse}

\section{Résumé}

Contexte : Le tabagisme par cigarettes est un facteur de risque partagé pour un grand nombre de maladies.

Objectifs : Estimer la prévalence du tabagisme chez les adolescents et les jeunes adultes iraniens.

Méthodes: Dans la présente revue systématique et méta-analyse, nous avons effectué des recherches dans PubMed, Web of Science, Embase, Scopus et des bases de données nationales de janvier 2001 à décembre 2018. Les études transversales rapportant la prévalence du tabagisme par cigarettes chez les lycéens, les étudiants ou dans la population générale âgée de 14 à 30 ans étaient éligibles. Des données sur la prévalence du tabagisme, l'âge et le sexe des sujets, la taille de l'échantillon, la date et le lieu des études ont été extraites par deux analystes indépendants.

Résultat: Nous avons inclus 99 études dans notre analyse. Pour les lycéens, la prévalence globale vie entière du tabagisme était de $13 \%$ chez les filles et de $23 \%$ chez les garçons. Pour les étudiants universitaires, la prévalence vie entière était de $11 \%$ chez les filles et de $33 \%$ chez les garçons. La prévalence cumulée du tabagisme actuel et régulier chez les garçons du secondaire était de $9 \%$ et $5 \%$, respectivement, contre $3 \%$ et $1 \%$ chez les filles. La prévalence cumulée du tabagisme actuel et régulier chez les étudiants universitaires de sexe masculin était de $22 \%$ et $11 \%$, respectivement, contre $5 \%$ et $1 \%$ chez les étudiantes. La prévalence du tabagisme actuel dans la population générale âgée de 15 à 24 ans était comprise entre 3,3\% et 13,9\%, La prévalence du tabagisme régulier au sein de cette population était de $1,0 \%$ à $10,2 \%$.

Conclusion : Le tabagisme par cigarettes est très répandu parmi les jeunes adultes iraniens, en particulier les garçons et les étudiants. Nos conclusions appellent au renforcement des politiques de prévention et de lutte antitabac dans l'ensemble des écoles et des universités iraniennes. 


\section{انتشار تدخين السجائر بين المر اهقين والشباب في إيران: استعر اض منهجي وتحليل تَكَوي \\ محمد الر اية، حميد شريفي، زهرة خور امي، غلايول أردلان، ليلى رجائي، غلام رضا حيدري، أرميتا شاه إسماعيل}

الخلفية: يُعتبر تدخين السجائر من عوامل الخطر المشتركة لمجموعة من الحالات المبل المرضية، مثل أمراض القلب والأوعية الدموية وبعض أنواع

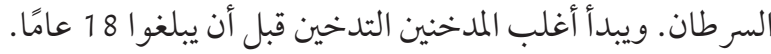

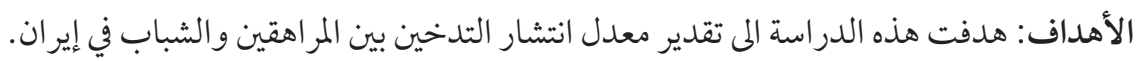

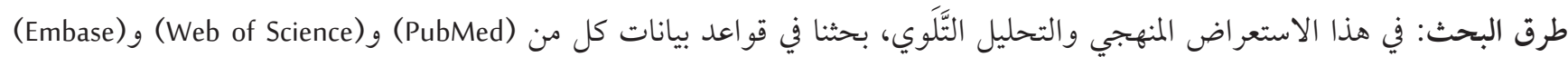

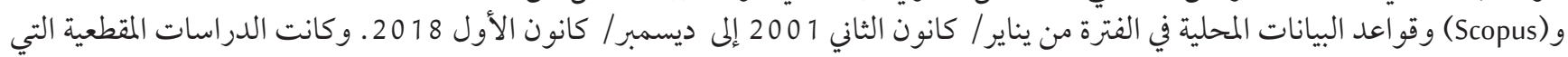

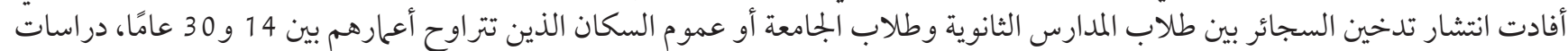

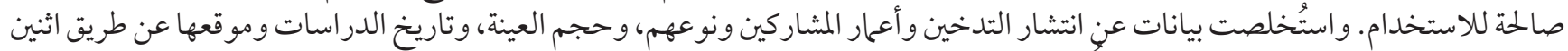

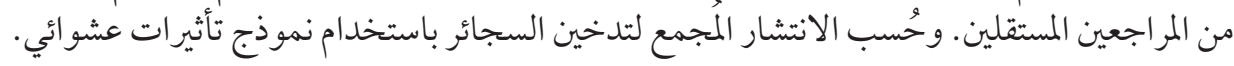

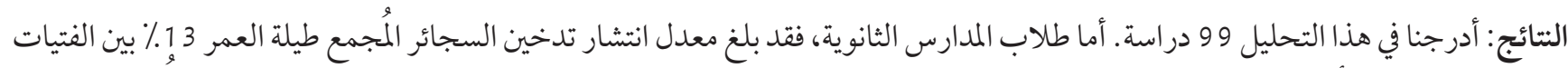

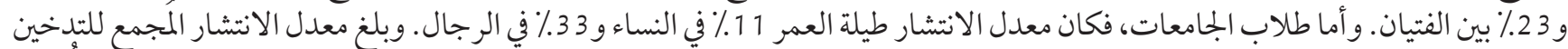

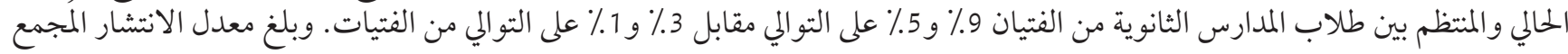

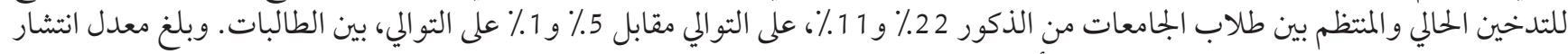

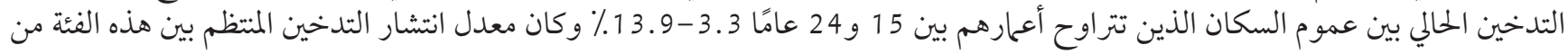

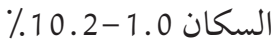

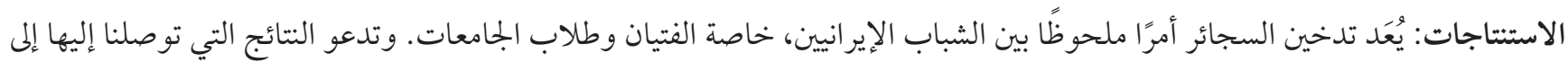

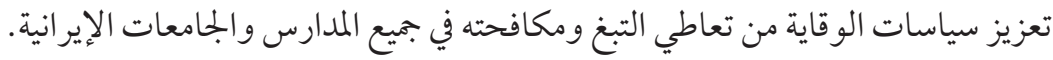

\section{References}

1. WHO report on the global tobacco epidemic 2017. Geneva: World Health Organization; 2017 (https://wwwwhoint/tobacco/global_report/en/, accessed 27 February 2021).

2. Goodchild M, Nargis N, d'Espaignet ET. Global economic cost of smoking-attributable diseases. Tob Control. 2018 Jan;27(1):5864. http://doi.org/10.1136/tobaccocontrol-2016-053305 PMID:28138063

3. Global status report on noncommunicable diseases. Geneva: World Health Organization; 2014 (https://wwwwhoint/nmh/publications/ncd-status-report-2014/en/, accessed 27 February 2021).

4. Chang CM, Corey CG, Rostron BL, Apelberg BJ. Systematic review of cigar smoking and all cause and smoking related mortality. BMC Public Health 2015 Apr 24;15:390. https://doi.org/10.1186/s12889-015-1617-5 PMID:25907101

5. Park S-H. Smoking and adolescent health. Korean J Pediatr. 2011 Oct;54(10):401-4. https://doi.org/10.3345/kjp.2011.54.10.401 PMID:22232621

6. Ayatollahi SA, Rajaeifard A, Mohammadpoorasl A. Predicting the stages of smoking acquisition in the male students of Shiraz's high schools, 2003. Nicot Tob Res. 2005 Dec 1;7(6):845-51. https://doi.org/10.1080/14622200500330233

7. Dowdell EB, Santucci ME. Health risk behavior assessment: nutrition, weight, and tobacco use in one urban seventh $\square$ grade class. Public Health Nurs. 2004 Mar-Apr;21(2):128-36. https://doi.org/10.1111/j.0737-1209.2004.021206.x PMID:14987212

8. Tamim H, Terro A, Kassem H, Ghazi A, Khamis TA, Hay MMA, et al. Tobacco use by university students, Lebanon, 2001. Addiction 2003 Jul;98(7):933-9. https://doi.org/10.1046/j.1360-0443.2003.00413.x PMID:12814499

9. Cullen J, Mowery P, Delnevo C, Allen JA, Sokol N, Byron MJ, et al. Seven-year patterns in US cigar use epidemiology among youths aged 18-25 years: a focus on race/ethnicity and brand. Am J Public Health. 2011 Oct;101(10):1955-62. https://doi. org/10.2105/AJPH.2011.300209 PMID:21852638

10. Arrazola RA, Dube SR, King BA. Tobacco product use among middle and high school students - United States, 2011 and 2012. MMWR Morb Mortal Wkly Rep. 2013 Nov 15;62(45):893. https://www.cdc.gov/mmwr/preview/mmwrhtml/mm6245a2.htm

11. Xi B, Liang Y, Liu Y, Yan Y, Zhao M, Ma C, et al. Tobacco use and second-hand smoke exposure in young adolescents aged 12-15 years: data from 68 low-income and middle-income countries. Lancet Global Health. 2016 Nov;4(11):e795-805. https://doi. org/10.1016/S2214-109X(16)30187-5 PMID:27697484

12. Rezaei F, Noroozi M, Mansourian M, Safari O, Jahangiry L. The role of social and familial factors as predicting factors related to hookah and cigarette smoking among adolescents in Jahrom, South of Iran. Int J Pediatr. 2017 May;5(5):4929-37. https://doi. org/10.22038/IJP.2017.22101.1850 
13. Roohafza H, Heidari K, Alinia T, Omidi R, Sadeghi M, Andalib E, et al. Smoking motivators are different among cigarette and waterpipe smokers: the results of ITUPP. J Epidemiol Glob Health 2015 Sep;5(3):249-58. https://doi.org/10.1016/j.jegh.2014.12.003 PMID:26231400

14. Ahmadi J, Hasani M. Prevalence of substance use among Iranian high school students. Addict Behav. 2003 March;28(2):375-9. https://doi.org/10.1016/s0306-4603(01)00246-5 PMID:12573688

15. Rad MHR, Rezaei MK, Abdollahi N, Hatami K. Smoking and asthma symptoms among university students. Tanaffos 2007;6(1):53-8.

16. Shamsi Meymandi M, Nakhaee N, Divsalar K, Heravi G. Cigarette smoking among Iranian university students: reasons and attitudes. Iranian J Psychiatry Behav Sci. 2010;4(2):37-41.

17. Hunter JP, Saratzis A, Sutton AJ, Boucher RH, Sayers RD, Bown MJ. In meta-analyses of proportion studies, funnel plots were found to be an inaccurate method of assessing publication bias. J Clin Epidemiol. 2014 Aug;67(8):897-903. https://doi. org/10.1016/j.jclinepi.2014.03.003 PMID:24794697

18. Mohtasham Amiri Z, Cirus Bakht S, Nikravesh Rad SR. Cigarette smoking among male high school students in Rasht. J Guilan Univ Med Sci. 2008 Apr 15;17(65):100-7. http://journal.gums.ac.ir/browse.php?a_id=333\&sid=1\&slc_lang=en

19. Heydari GR, Sharifi MH, Hosseini M, Masjedi MR. Attitude of high school students of Tehran towards tobacco use. Tanaffos 2004:3(11):29-35. https://www.sid.ir/en/journal/ViewPaper.aspx?id=45450

20. Karimi M, Kaveh MH, Morowatisharifabad MA, Dehghani A, Dastjerdi G. Cigarette smoking experience and its related socio-demographic and environmental risk factors in high school boy students, Shiraz - Iran. Int J Pediatr. 2017 Feb;5(2):4263-74. https:// doi.org/10.22038/IJP.2017.21122.1773

21. Heydari G, Yousefifard M, Hosseini M, Ramezankhani A, Masjedi MR. Cigarette smoking, knowledge, attitude and prediction of smoking between male students, teachers and clergymen in tehran, iran, 2009. In J Prev Med. 2013 May;4(5):557-64. PMID:23930167

22. Habib E, Shiraz ASR, Naseri-Kouzehgarani G, Hooman S, Reza MM. The determinants of high school students smoking habits with special focus on teachers smoking in Iran: a population based study. Pneumologia 2012 Jan-Mar;61(1):28-33. PMID:22545486

23. Mohammadi F, Zakarianejad M, Valizadeh F. Determinants of smoking behavior among male high school students in Babolsar, Iran. J Mazandaran Univ Med Sci. 2014;24(116):216-22.

24. Rahmanian K, Jafarzadeh A, Khalooei A. [Determinants of smoking behavior among high school students in Jahrom]. Payavard Salamat 2010;4(2):88-96 (in Farsi).

25. Pirdehghan A, Rezaee MP, Mirzababaee B. Epidemiology of substance abuse among Iranian adolescents (Yazd: 2014). Iran J Psychiatry Behav Sci. 2017;11(4):e3743. https://doi.org/10.5812/ijpbs.3743

26. Vafaei B, SHahamfar j, Fadaei hagh A. The causes of smoking tendency and the effect of two methods of counseling, guidance, psychotherapy, and nicotine gum on smoking. Addiction Journal of Faculty of Medicine, Tehran University of Medical Sciences 2004;62(10):877-84 (in Persian).

27. Reisi M, Javadzade SH, Shahnazi H, Sharifirad G, Charkazi A, Moodi M. Factors affecting cigarette smoking based on health-belief model structures in pre-university students in Isfahan. J Educ Health Promot. 2014 Feb 21;3:23. http://doi.org/10.4103/22779531.127614 PMID:24741663

28. Mozafarinia R, Assarian M, Ziaaddini A. Prevalence of substance abuse among students of Tehran University of Medical Sciences, Iran. Addiction Health. 2017 Apr;9(2):103-9. PMID:29299213

29. Sabzmakan L, Ghasemi M, Asghari Jafarabadi M, Kamalikhah T, Chaleshgar Kordasiabi M. Factors associated with tobacco use among Iranian adolescents: an application of protection motivation theory. Subst Use Misuse 2018 Jul 29;53(9):1511-8. http://doi. org/10.1080/10826084.2017.1415356 PMID:29313745

30. Meysamie A, Seddigh L, Mahdiin Z. Frequency of tobacco use among students in Tehran city. Tehran Univ Med J. 2015 Oct;73(7):515-26. http://tumj.tums.ac.ir/article-1-6913-en.html

31. Karimy M, Niknami S, Hidarnia AR, Hajizadeh I. Intention to start cigarette smoking among Iranian male adolescents: usefulness of an extended version of the theory of planned behaviour. Heart Asia 2014 Sep 26;4(1):120-4. http://doi.org/10.1136/heartasia-2012-010140 PMID:27326046

32. Karimy M, Niknami S, Heidarnia AR, Hajizadeh E, Shamsi M. Predicting smoking among adolescents: examining the role of smoking self-identity in the theory of planned behavior. Health Syst Res. 2013 Jun;9(3):301-11.

33. Mohammadi S, Ghajari H, Valizade R, Ghaderi N, Yousefi F, Taymoori P, et al. Predictors of smoking among the secondary high school boy students based on the health belief model. Int J Prev Med. 2017 Apr 13;8:24. http://doi.org/10.4103/ijpvm.IJPVM_264_16 PMID:28479966

34. Barati M, Niknami S, Hidarnia A, Allahverdipour H. Predictors of tobacco smoking in male adolescents in Hamadan based on the theory of planned behavior. J Educ Community Health 2014 Autumn;1(3):28-37. http://jech.umsha.ac.ir/article-1-64-en.html

35. Sahebihagh MH, Hajizadeh M, Ansari H, Lesani A, Fakhari A, Mohammadpoorasl A. Modeling the underlying tobacco smoking predictors among 1(st) year university students in Iran. Int J Prev Med. 2017 Nov 7;8:90. https://doi.org/10.4103/ijpvm. IJPVM_311_16 PMID:29184641 
36. Moeini B, Poorolajal J, Gharghani ZG. Prevalence of cigarette smoking and associated risk factors among adolescents in Hamadan City, west of Iran in 2010. J Res Health Sci. 2012;12(1):31-7. PMID:22888712

37. Namakin K, Sharifzadeh G, Miri M. Prevalence of cigarette smoking and evaluation of attitude and knowledge in its high school boys in Birjand 2005. J Birjand Univ Med Sci. 2008 Spring;15(1):66-70. http://journal.bums.ac.ir/article-1-263-en.html

38. Mohamadkhani S. Prevalence of cigarette smoking, alcohol drinking and illegal drugs use among Iranian adolescents. J Kerman Univ Med Sci. 2011;18(1):32-42.

39. Mohammadkhani S. Prevalence of cigarette smoking, alcohol drinking and illegal drugs use among Iranian adolescents. J Kerman Univ Med Sci. 2012;19(1):32-48.

40. Shamshiri Milani H, Abadi AR, Helmzadeh Z, Abachizadeh K. Prevalence of Ecstasy use and predisposing factors among Iranian female high school students. J Pak Med Assoc. 2011 Jun;61(6):566-71. PMID:22204212

41. Heydari G, Sharifi H, Hosseini M, Masjedi MR. Prevalence of smoking among high-school students of Tehran in 2003. East Mediterr Health J. 2007 Sep-Oct;13(5):1017-21. https://doi.org/10.26719/2007.13.5.1017 PMID:18290393

42. Mojahed A, Bakhshani N. [Prevalence of smoking and drug abuse in students of Zahedan high schools]. Zahedan J Res Med Sci. 2004;6(1):e95038 (in Farsi).

43. Pasharoosh L, khoshbo S, Rezaei M, Saeidi M. Prevalence of smoking and associated factors in female high school in Kermanshah. J Kermanshah Univ Med Sci. 2009;13(4):309-19.

44. Ramezankhani A, Zaboli FS, Zarghi A, Masjedi MR, Heydari GR. Smoking habits of adolescent students in Tehran. Tanaffos 2010;9(2):33-42.

45. Ataeiasl M, Sarbakhsh P, Dadashzadeh H, Augner C, Anbarlouei M, Mohammadpoorasl A. Relationship between happiness and tobacco smoking among high school students. Epidemiol Health. 2018 Mar 24;40:e2018009. https://doi.org/10.4178/epih.e2018009 PMID:29587337

46. Bashirian S, Barati M, Abasi H, Sharma M, Karami M. The role of sociodemographic factors associated with water pipe smoking among male adolescents in western Iran: a cross-sectional study Tob Induc Dis. 2018 Jun 5;16:29. https://doi.org/10.18332/ tid/91601 PMID:31516429

47. Khajehdaluee M, Zavar A, Alidoust M, Pourandi R. Smoking among high school students of an area with medium socioeconomic status. J Pak Med Assoc. 2014 Mar;64(3):291-5. PMID:24864602

48. Chaman R, Khosravi A, Sajedinejad S, Nazemi S, Fereidoon Mohasseli K, Valizade B, et al. Smoking and its related factors among Iranian high school students. Iran J Psychiatry Behav Sci. 2015 Dec;9(4):e1583. http://doi.org/10.17795/ijpbs-1583 PMID:26834798

49. Pirdehghan A, Vakili M, Arab M, Aghakoochak A. [Smoking frequency and modeling the underlying predicting factors of tobacco smoking among high school students in Yazd city, 2012]. J Shahrekord Univ Med Sci. 2014;16(5)56-65 (in Farsi). http://journal. skums.ac.ir/article-1-1820-en.html

50. Mohammadpoorasl A, Nedjat S, Fakhari A, Yazdani K, Rahimi Foroushani A, Fotouhi A. Smoking stages in an Iranian adolescent population. Acta Med Iran. 2012;50(11):746-54. PMID:23292626

51. Bidel Z, Nazarzadeh M, SaburMohamadi M, Zareimanesh E, Tazval J, Mohamadi E, et al. Smoking stages, prevalence of drug abuse and role of associated psychological and social factors: A study on male high school students in Ilam city. J Kerman Univ Med Sci. 2014 Feb-Mar;21(1):81-93.

52. Nazarzadeh M, Bidel Z, Ayubi E, Bahrami A, Jafari F, Mohammadpoorasl A, et al. Smoking status in Iranian male adolescents: a cross-sectional study and a meta-analysis. Addict Behav. 2013 Jun;38(6):2214-8. https://doi.org/10.1016/j.addbeh.2013.01.018 PMID:23501137

53. Khayat S, Dolatian M, Navidian A, Mahmoodi Z, Sharifi N, Kasaeian A. Lifestyles in suburban populations: a systematic review. Electron Physician. 2017 Jul 25;9(7):4791-800. https://doi.org/10.19082/4791 PMID:28894537

54. Yazdani R, Vehkalahti MM, Nouri M, Murtomaa H. Smoking, tooth brushing and oral cleanliness among 15-year-olds in Tehran, Iran. Oral Health Prev Dent. 2008;6(1):45-51. PMID:18399307

55. Ayubi E, Sani M, Safiri S, Morasae EK, Almasi-Hashiani A, Nazarzadeh M. Socioeconomic determinants of inequality in smoking stages: a distributive analysis on a sample of male high school students. Am J Mens Health 2017 Jul;11(4):1162-8. https://doi. org/10.1177/1557988315585822 PMID:26008735

56. Ziaee P, Hatamizadeh N, Vameghi R, Dolatabadi SH. A study on prevalence of cigarette smoking and the age of first smoking in senior high school students in Tehran, 1998-1999. Hakim Res J. 2001;2:78-84.

57. Khayyati F, Mohammadpoorasl A, Allahverdipour H, AsghariJafarabadi M, Kouzekanani K. Subgrouping high school students for substance abuse-related behaviors: a latent class analysis. Am J Mens Health. 2017 Jul;11(4):1200-7. https://doi. org/10.1177/1557988316669046 PMID:27694553

58. Momtazi S, Rawson RA. Substance abuse among Iranian high school students. Curr Opin Psychiatry. 2010 May;23(3):221. https:// doi.org/10.1097/YCO.obo13e328338630d PMID:20308905

59. Mohammad Poorasl A, Vahidi R, Fakhari A, Rostami F, Dastghiri S. Substance abuse in Iranian high school students. Addict Behav. 2007 Mar;32(3):622-7. https://doi.org/10.1016/j.addbeh.2006.05.008 PMID:16815638

6o. Poorolajal J, Ghaleiha A, Darvishi N, Daryaei S, Panahi S. The prevalence of psychiatric distress and associated risk factors among college students using GHQ-28 questionnaire. Iranian J Public Health. 2017 Jul;46(7):957-63. PMID:28845407 
61. Taheri E, Ghorbani A, Salehi M, Sadeghnia HR. Cigarette smoking behavior and the related factors among the students of mashhad university of medical sciences in iran. Iran Red Crescent Med J. 2015 Dec 27;17(1):e16769. https://doi.org/10.5812/ircmj.16769 PMID:25763254

62. Kassiri H, Rafiee A, Haghighizadeh MH, Kazemzadeh N. Epidemilogy of cigarette smoking among male students of Ahvaz Jundishapur University of Medical Sciences, Iran. Jentashapir J Cell Mol Biol. 2011 Summer;2(2):75-84.

63. Shoja M, Jouybari L, Ghorbani M, Sanagou A, SHojaei H, Kiakajouri Z, et al. Prevalence and cause of smoking among the dormitories students in Gorgan University of Medical Sciences. Researcher Bull Med Sci. 2010 Aug-Sep;15(3):123-8.

64. Machowicz R, Ciechanska J, Zycinska K, Mahboobi N, Wnekowicz E, Obrowski MH, et al. Medical students' aptitude toward smoking in Warsaw, Strasbourg and Teheran. Adv Exp Med Biol. 2013;755:195-202.

65. Jamali A, Tofangchiha S, Jamali R, Nedjat S, Jan D, Narimani A, et al. Medical students' health-related quality of life: roles of social and behavioural factors Med Educ. 2013;47(10):1001-12. https://doi.org/10.1111/medu.12247 PMID:24016170

66. Sahebihagh MH, Hajizadeh M, Ansari H, Lesani A, Fakhari A, Mohammadpoorasl A. Modeling the underlying tobacco smoking predictors among 1st year university students in Iran. Int J Prev Med. 2017 Nov 7;8:90. https://doi.org/10.4103/ijpvm. IJPVM_311_16 PMID:29184641

67. Nojomi M, Najamabadi S. Obesity among university students, Tehran, Iran. Asia Pac J Clin Nutr 2006;15(4):516-20. PMID:17077068

68. Abbasi-Ghahramanloo A, Rahimi-Movaghar A, Zeraati H, Fotouhi A. Pattern of substance use among students of medical sciences in Tehran, Iran: a latent class analysis. J Subst Use. 2018 Nov 2;23(6):648-54. https://doi.org/10.1080/14659891.2018.1489007

69. Ghodousi A, Aminoroaia M, Attari A, Maracy M, Maghsoodloo S. The prevalance of cigarette smoking and some demographic and psychological characteristics in students of Islamic Azad University of Khorasgan. Iran J Res Behav Sci. 2013;10(6):401-10. http://rbs.mui.ac.ir/article-1-273-en.html

70. Majidpour A, Arbaby YH, Abbasgholizadeh N, Salehy E. Prevalence and causes of tendency to cigarette smoking among students of Ardabil University of Medical Sciences. J Ardabil Univ Med Sci. 2005;5(3):266-70. http://jarums.arums.ac.ir/article-1-591-en. html

71. Seghatoleslam T, West R, Agin K, Habil H, Seghatoleslam N, Ardakani A, et al. Prevalence and characterizations of current smoking habit of iranian medical university students: a cross-sectional study in mental health. Int Med J. 2013;20(3).

72. Ghodsi H, Mokhtari Lake N, Asiri S, Kazem Nezhad Leili E. Prevalence and correlates of cigarette smoking among male students of Guilan University of Medical Sciences. J Holist Nurs Midwifery 2012;22(1):38-43. http://hnmj.gums.ac.ir/article-1-177-en.html

73. Mohtasham-Amiri Z, Jafari-Shakib A, Khalili-Moosavi A. Prevalence and factors associated with Ecstasy use among college undergraduates in north of Iran - 2005. Asian J Psychiatry. 2011;4(1):31-4. https://doi.org/10.1016/j.ajp.2011.01.004 PMID:23050911

74. Nemati S, Rafei A, Freedman ND, Fotouhi A, Asgary F, Zendehdel K. Cigarette and water-pipe use in Iran: geographical distribution and time trends among the adult population; a pooled analysis of national STEPS Surveys, 2006-2009. Arch Iran Med. 2017 May;20(5):295-301. PMID:28510465

75. Amiri ZM, Shakib AJ, Moosavi AK. Prevalence and risk factors of ecstasy use among college students in Astara, Islamic Republic of Iran. East Mediterr Health J. 2009 Sep-Oct;15(5):1192-200. PMID:20214133

76. Mohammadpoorasl A, Abbasi Ghahramanloo A, Allahverdipour H, Modaresi Esfeh J. Prevalence of Hookah smoking in relation to religiosity and familial support in college students of Tabriz, northwest of Iran. J Res Health Sci. 2014 Autumn;14(4):268-71. PMID:25503281

77. Mohammadi S, Ghajari H, Valizade R, Ghaderi N, Yousefi F, Taymoori P, et al. Predictors of smoking among the secondary high school boy students based on the health belief model. Int J Prev Med. 2017 Apr 13;8:24. PMID:28479966

78. Shojaa M, Qhorbani M, Jouybari LM, Sanagoo A, Mohebi R, Bamyar R, et al. Prevalence of smoking among the students resided at dormitories in Golestan University of Medical Sciences, Iran. Bangladesh J Med Sci. 2014;13(4):460-5. https://doi.org/10.3329/ bjms.v13i4.16727

79. Tavakkolizadeh J, Ghahramani M, Moghimian M. The survey of stressful events on smoked and nonsmoked early adults of Gonabad city. Horizon Med Sci. 2004 Apr 15;10(1):52-60. http://hms.gmu.ac.ir/article-1-260-en.html

8o. Heydarabadi AB, Ramezankhani A, Barekati H, Vejdani M, Shariatinejad K, Panahi R, et al. Prevalence of substance abuse among dormitory students of Shahid Beheshti University of Medical Sciences, Tehran. Iran Int J High Risk Behav Addict. 2015 Jun 20;4(2):e22350. https://doi.org/10.5812/ijhrba.22350v2 PMID:26405679

81. Shuja M, Sarrafzadegan N, Roohafza HR, Sadeghi M, Ghafari M, Mohammadian M, et al. Factors associated with cigarette smoking in central parts of Iran. Asian Pac J Cancer Prev. 2017 Mar 1;18(3):647-53. https://doi.org/10.22034/APJCP.2017.18.3.647 PMID:28440970

82. Fajani S, Janghorbani M, Khosravi A. [Prevalence of substance abuse and its association with cigarette smoking in isfahan university of medical sciences students in 2013]. Koomesh 2015;16(4):544-54 (in Farsi). http://koomeshjournal.semums.ac.ir/article1-2516-en.html

83. Nakhaee N, Divsalar K, Jadidi N. Religious involvement and cigarette smoking among Iranian university students Int J Psychiatry Med. 2009;39(2):189-98. https://doi.org/10.2190/PM.39.2.g PMID:19860077 
84. Jafari F, Haji Zamani A, Alizadeh K. Reviewing the prevalence of (cigarette) smoking and its related factors in students of tehran university, iran Addict Health. 2011 Summer-Autumn;3(3-4):105-10. PMID:24494124

85. Mohammadpoorasl A, Ghahramanloo AA, Allahverdipour H. Risk-taking behaviors and subgrouping of college students: a latent class analysis. Am J Mens Health 2013 Nov;7(6):475-81. https://doi.org/10.1177/1557988313483540 PMID:23539632

86. Zahedi R, Noroozi A, Hajebi A, Haghdoost AA, Baneshi MR, Sharifi H, Mirzazadeh A. Self-reported and network scale-up estimates of substance use prevalence among university students in Kerman, Iran. J Res Health Sci. 2018 Apr 30;18(2):eo0413. PMID:29784894

87. Nazary AA, Ahmadi F, Vaismoradi M, Kaviani K, Arezomandi M, Faghihzadeh S. Smoking among male medical sciences students in Semnan, Islamic Republic of Iran. East Mediterr Health J. 2010 Feb;16(2):156-61. PMID:20799567

88. Rahimi Rad MH, Rezaei MK, Abdollahi N, Hatami K. Smoking and asthma symptoms among university students. Tanaffos. 2007;6(1):53-8.

89. Nasirian M, Ziaaddini H, Asadollahi S. Smoking intensity and its relation to general health of the students of kerman university of medical sciences, iran. Addict Health. 2013 Summer-Autumn;5(3-4):102-7. PMID:24494166

90. Menati W, Nazarzadeh M, Bidel Z, Wurtz M, Menati R, Hemati R, et al. Social and psychological predictors of initial cigarette smoking experience: a survey in male college students. Am J Mens Health 2016 Jan;10(1):14-23. https://doi. org/10.1177/1557988314553262 PMID:25326133

91. Jalilian F, Karami Matin B, Ahmadpanah M, Ataee M, Ahmadi Jouybari T, Eslami AA, et al. Socio-demographic characteristics associated with cigarettes smoking, drug abuse and alcohol drinking among male medical university students in Iran. J Res Health Sci. 2015 Winter;15(1):42-6. PMID:25821025

92. Tarrahi MJ, Mohammadpoorasl A, Ansari H, Mohammadi Y. Substance Abuse and Its Predictors in Freshmen Students of Lorestan Universities: subgrouping of college students in west of Iran. Health Scope. 2017;6(4):e64186. 10.5812/jhealthscope. 64186

93. Ahmadi J, Maharlooy N, Alishahi M. Substance abuse: prevalence in a sample of nursing students. J Clin Nurs. 2004 Jan;13(1):604. https://doi.org/10.1046/j.1365-2702.2003.00841.x PMID:14687294

94. Zarrabi H, Najafi K, Kafi M, Shirazi M. Substance use among students of Guilan University of Medical Sciences in Iran in 2005-2006. Acta Medica Iranica 2009;47(6):473-8. https://acta.tums.ac.ir/index.php/acta/article/view/3632

95. Kabir K, Mohammadpoorasl A, Esmaeelpour R, Aghazamani F, Rostami F. Tobacco use and substance abuse in students of Karaj Universities. Int J Prev Med. 2016 Sep 8;7:105. https://doi.org/10.4103/2008-7802.190091 PMID:27688869

96. Amin-Esmaeili M, Rahimi-Movaghar A, Yunesian M, Sahimi-Izadian E, Moinolghorabaei M. Trend of smoking among students of Tehran University of Medical Sciences: results from four consecutive surveys from 2006 to 2009. Med J Islam Repub Iran. 2013 Nov;27(4):168-78. PMID:24926177

97. Mosavi-Jarrahi A, Mohagheghi M, Yazdizadeh B, Kolahi AA, Tahmasebi S, Sharifi S. Analysis of smoking behaviour among Iranian population: a cohort and period analysis. Asian Pac J Cancer Prev. 2004 Jan-Mar;5(1):66-9. PMID:15075008

98. Abdollahifard G, Vakili V, Danaei M, Askarian M, Romito L, Palenik CJ. Are the predictors of hookah smoking differ from those of cigarette smoking? Report of a population-based study in Shiraz, Iran, 2010. Int J Prev Med. 2013;4(4):459-66. PMID:23671779

99. Nekoei Moghadam M, Ahmadizadeh Fini E, Khanjani N, Vali L, Rafiezad E, Karim Dashtiyan M, et al. Assessment of non-communicable disease risk factors in hormozgan province Life Sci J. 2013;10(12S):405-8.

100. Hoseini M, Yunesian M, Nabizadeh R, Yaghmaeian K, Parmy S, Gharibi H, et al. Biomonitoring of tobacco smoke exposure and self-reported smoking status among general population of Tehran, Iran. Environ Sci Pollut Res Int. 2016 Dec;23(24):25065-73. https://doi.org/10.1007/s11356-016-7619-8 PMID:27677995

101. Sadeghi M, Roohafza HR, Kelishadi R. Blood pressure and associated cardiovascular risk factors in Iran: Isfahan Healthy Heart Programme. Med J Malaysia. 2004 Oct;59(4):460-7. PMID:15779578

102. Hassanzadeh J, Asadi-Lari M, Ghaem H, Kassani A, Rezaianzadeh A. Demographic factors, social capital, and cigarette smoking: a large cross-sectional study in Tehran, Iran. J Subst Use. 2016;21(6):581-6. https://doi.org/10.3109/14659891.2015.1112850

103. Moghimbeigi A, Eshraghian MR, Mohammad K, Nourijelyani K, Husseini M. Determinants number of cigarette smoked with Iranian adolescents: A multilevel zero inflated poisson regression model. Iran J Public Health. 2009;38(4):91-6.

104. Shuja M, Sarrafzadegan N, Roohafza HR, Sadeghi M, Ghafari M, Mohammadian M, et al. Factors associated with cigarette smoking in central parts of Iran. Asian Pac J Cancer Prev. 2017 Mar 1;18(3):647-53. https;//doi.org/10.22034/APJCP.2017.18.3.647 PMID:28440970

105. Meysamie A, Ghaletaki R, Haghazali M, Asgari F, Rashidi A, Khalilzadeh O, et al. Pattern of tobacco use among the Iranian adult population: results of the national Survey of Risk Factors of Non-Communicable Diseases (SuRFNCD-2007) Tob Control. 2010 Apr;19(2):125-8. https://doi.org/10.1136/tc.2009.030759 PMID:20008159

106. Baheiraei A, Hamzehgardeshi Z, Mohammadi MR, Nedjat S, Mohammadi E. Personal and family factors affecting life-timecigarette smoking among adolescents in Tehran (Iran): a community based study. Oman Med J. 2013 May;28(3):184-90. https://doi. org/10.5001/omj.2013.51 PMID:23772284

107. Baheiraei A, Mirghafourvand M, Charandabi SMA, Nedjat S, Mohammadi E. A population-based survey on prevalence of cigarette smoking and its socio-demographic risk factors among women of reproductive age in Tehran - Iran. Epidemiol Biostat Public Health. 2014;11(3):e9408-1-e-8. 
108. Salimzadeh H, Najafipour H, Mirzaiepour F, Navadeh S, Shadkam-Farrokhi M, Mirzazadeh A. Prevalence of active and passive smoking among adult population: findings of a population-based survey in Kerman (KERCADRS), Iran. Addict Health. 2016 Winter;8(1):16-24. PMID:27274789

109. Kassani A, Baghbanian A, Menati R, Hassanzadeh J, Asadi-Lari M, Menati W. Prevalence of cigarette smoking and associated factors among male citizens in Tehran, Iran. Asian Pac J Cancer Prev. 2016;17(3):1473-8. https://doi.org/10.7314/apjcp.2016.17.3.1473 PMID:27039792

110. Fotouhi A, Khabazkhoob M, Hashemi H, Mohammad K. The prevalence of cigarette smoking in residents of Tehran. Arch Iran Med. 2009 Jul;12(4):358-64. PMID:19566352

111. Ali EN, Jafar A. Prevalence of cigarette smoking in the Rafsanjan urban population. East Mediterr Health J. 2009 JulAug;15(4):1032-5. PMID:20187557

112. Hoseini M, Yunesian M, Nabizadeh R, Yaghmaeian K, Parmy S, Gharibi H, et al. Biomonitoring of tobacco smoke exposure and self-reported smoking status among general population of Tehran, Iran. Environl Sci Pollut Res Int. 2016 Dec;23(24):25065-73. https://doi.org/10.1007/s11356-016-7619-8 PMID:27677995

113. Haghdoost AA, Moosazadeh M. The prevalence of cigarette smoking among students of Iran's universities: a systematic review and meta-analysis. J Res Med Sci. 2013 Aug;18(8):717-25. PMID:24379851

114. Kruger L, van Walbeek C, Vellios N. Waterpipe and cigarette smoking among university students in the Western Cape, South Africa. Am J Health Behav. 2016 Jul;40(4):416-26. https://doi.org/10.5993/AJHB.40.4.3 PMID:27338988

115. Higgins ST, Kurti AN, Redner R, White TJ, Gaalema DE, Roberts ME, et al. A literature review on prevalence of gender differences and intersections with other vulnerabilities to tobacco use in the United States, 2004-2014. Prev Med. 2015 Nov;80:89-100. https://doi.org/10.1016/j.ypmed.2015.06.009 PMID:26123717

116. Rafique I, Saqib MA, Munir MA, Qureshi H, Rizwanullah KS, Khan SA, et al. Prevalence of risk factors for noncommunicable diseases in adults: key findings from the Pakistan STEPS survey. East Mediterr Health J. 2018 Apr 5;24(1):33-41. PMID:29658619

117. Tucktuck M, Ghandour R, Abu-Rmeileh NME. Waterpipe and cigarette tobacco smoking among Palestinian university students: a cross-sectional study BMC Public Health. 2017;18:1. https://doi.org/10.1186/s12889-017-4524-0

118. Idris A, Al Saadi T, Turk T, Alkhatib M, Zakaria M, Sawaf B, et al. Smoking behaviour and patterns among university students during the Syrian crisis. East Mediterr Health J. 2018 May 3;24(2):154-60. PMID:29748944

119. Sreeramareddy CT, Ramakrishnareddy N, Rahman M, Mir IA. Prevalence of tobacco use and perceptions of student health professionals about cessation training: results from Global Health Professions Students Survey. BMJ Open 2018 May 26;8(5):e017477. https://doi.org/10.1136/bmjopen-2017-017477 PMID:29804056

120. Sabahy A, Divsalar K, Bahreinifar S, Marzban M, Nakhaee N. Waterpipe tobacco use among Iranian university students: correlates and perceived reasons for use Tint J Tuberc Lung Dis. 2011 Jun;15(6):844-7. https://doi.org/10.5588/ijtld.10.0744 PMID:21575309

121. Ghafouri N, Hirsch JD, Heydari G, Morello CM, Kuo GM, Singh RF. Waterpipe smoking among health sciences university students in Iran: perceptions, practices and patterns of use. BMC Research Notes 2011 Nov 16;4(1):496. https://doi.org/10.1186/17560500-4-496 PMID:22087840

122. Salimzadeh H, Najafipour H, Mirzaiepour F, Navadeh S, Shadkam-Farrokhi M, Mirzazadeh A. Prevalence of active and passive smoking among adult population: findings of a population-based survey in Kerman (KERCADRS), Iran. Addict Health. 2016 Winter;8(1):16-24. PMID:27274789

123. Abachizadeh K, Ekhtiari YS, Kolahi A-A. Smoking pattern and associated sociodemographic factors: findings from a nationwide STEPS survey in Iran. Int J Prev Med. 2018 Dec 5;9:105. https://doi.org/10.4103/ijpvm.IJPVM_488_17 PMID:30622688

124. Parizadeh D, Momenan AA, Amouzegar A, Azizi F, Hadaegh F. Tobacco smoking: findings from 20 years of the Tehran Lipid and Glucose Study. Int J Endocrinol Metab. 2018 Oct 31;16(4 Suppl):e84738. https://doi.org/10.5812/ijem.84738 PMID:30584430

125. Ministry of Science, Research and Technology, Islamic Republic of Iran. Statistics - 2019 [website] (https://www.msrt.ir/en/ page/20/statistics-2019\#Universities, accessed 3 March 2021).

126. Hitchman SC, Fong GT. Gender empowerment and female-to-male smoking prevalence ratios. Bull WHO. 2011;89:195-202. http://dx.doi.org/10.2471/BLT.10.079905

127. Barengolts E, Green SJ, Eisenberg Y, Akbar A, Reddivari B, Layden BT, et al. Gut microbiota varies by opioid use, circulating leptin and oxytocin in African American men with diabetes and high burden of chronic disease. PLoS One. 2018 Mar 29;13(3):e0194171. https://doi.org/10.1371/journal.pone.0194171 PMID:29596446

128. El-Shahawy O, Nicksic NE, Ramôa C, Jawad M, Niaura R, Abrams D, et al. Linking Global Youth Tobacco Survey Data to the WHO Framework Convention on Tobacco Control: the case for Egypt. Curr Addict Rep. 2018;5(1):54-64. https://doi.org/10.1007/ s40429-018-0184-0

129. WHO report on the global tobacco epidemic, 201.7 Country profile Iran (Islamic Republic of). Geneva: World Health Organization; 2017 (http://wwwwhoint/tobacco/surveillance/policy/country_ profile/irnpdf,accessed 2 August 2019). 